\title{
An Efficient Approach to Numerical Study of the MRLW Equation with B-Spline Collocation Method
}

\author{
Seydi Battal Gazi Karakoç, ${ }^{1}$ Turgut Ak, ${ }^{2}$ and Halil Zeybek ${ }^{3}$ \\ ${ }^{1}$ Nevsehir Haci Bektas Veli University, 50300 Nevsehir, Turkey \\ ${ }^{2}$ Armutlu Vocational High School, Yalova University, 77500 Yalova, Turkey \\ ${ }^{3}$ Faculty of Computer Science, Abdullah Gül University, 38039 Kayseri, Turkey \\ Correspondence should be addressed to Seydi Battal Gazi Karakoç; sbgkarakoc@nevsehir.edu.tr \\ Received 1 January 2014; Revised 15 May 2014; Accepted 8 July 2014; Published 22 July 2014 \\ Academic Editor: Ahmet Yasar Ozban
}

Copyright (C) 2014 Seydi Battal Gazi Karakoç et al. This is an open access article distributed under the Creative Commons Attribution License, which permits unrestricted use, distribution, and reproduction in any medium, provided the original work is properly cited.

\begin{abstract}
A septic B-spline collocation method is implemented to find the numerical solution of the modified regularized long wave (MRLW) equation. Three test problems including the single soliton and interaction of two and three solitons are studied to validate the proposed method by calculating the error norms $L_{2}$ and $L_{\infty}$ and the invariants $I_{1}, I_{2}$, and $I_{3}$. Also, we have studied the Maxwellian initial condition pulse. The numerical results obtained by the method show that the present method is accurate and efficient. Results are compared with some earlier results given in the literature. A linear stability analysis of the method is also investigated.
\end{abstract}

\section{Introduction}

The generalized regularized long wave equation is given by

$$
U_{t}+U_{x}+\delta U^{p} U_{x}-\mu U_{x x t}=0
$$

where $p$ is a positive integer and $\delta$ and $\mu$ are positive constants. This equation is one of the most important nonlinear wave equation used a model for small amplitude long waves on the surface of water in a channel $[1,2]$. A few authors solved the equation numerically: among others, Zhang [3] used a finite difference method for a Cauchy problem and Kaya [4] applied the Adomian decomposition method and a quasilinearization method based on finite differences was used by Ramos [5]. Roshan [6] implemented the PetrovGalerkin method using a linear hat function as the trial function and a quintic B-spline function as the test function. A mesh-free technique for the numerical solution of the equation has been presented by Mokhtari and Mohammadi [7]. For $p=1$,

$$
U_{t}+U_{x}+\delta U U_{x}-\mu U_{x x t}=0
$$

Equation (2) is known as regularized long wave equation, originally introduced to describe the behavior of the undular bore by Peregrine [1] and later widely studied by Benjamin et al. [8]. The RLW equation has been solved numerically by finite element methods [9-22], finite difference methods [2326], Fourier pseudospectral [27], and mesh-free method [28]. For $p=2$,

$$
U_{t}+U_{x}+6 U^{2} U_{x}-\mu U_{x x t}=0 .
$$

Another particular case of (2) is called modified regularized long wave (MRLW) equation. Like RLW equation, the MRLW equation has been solved numerically by various methods. Among many others, a collocation solution to the equation using quintic B-spline finite element method is developed by Gardner et al. [29]. Khalifa et al. [30, 31] obtained the numerical solutions of the equation using finite difference method and cubic B-spline collocation finite element method. Solutions based on collocation method with quadratic B-spline finite elements and the central finite difference method for time are investigated by Raslan [32]. The equation was solved with a collocation finite element method using quadratic, cubic, quartic, and quintic B-splines to obtain the numerical 
solutions of the single solitary wave by Raslan and Hassan [33]. Haq et al. [34] have designed a numerical scheme based on quartic B-spline collocation method for the numerical solution of the equation. Ali [35] has formulated a classical radial basis functions (RBFs) collocation method for solving the equation. Karakoc et al. [36] have obtained a type of the quintic B-spline collocation procedure in which nonlinear term in the equation is linearized by using the form introduced by the Rubin and Graves [37] to solve the equation. A Petrov-Galerkin method using cubic B-spline function as trial function and a quadratic B-spline function as the test function is set up to solve the equation by Karakoc and Geyikli [38]. A homotopy analysis method has been employed to obtain approximate numerical solution of the modified regularized long wave (MRLW) equation with some specified initial conditions by Khan et al. [39].

In the present paper, a numerical scheme based on the septic B-spline collocation method has been set up for solving the MRLW equation with a variant of both initial and boundary conditions. This paper is set out as follows. In Section 2, septic B-spline collocation scheme is presented. Also stability analysis is considered. In Section 3, test problems including single, two, and three solitary waves and Maxwellian initial condition are discussed. Finally in Section 4, a summary is given at the end of the paper.

\section{Septic B-Spline Finite Element Solution}

Consider the MRLW Equation (3) given with the following boundary conditions,

$$
\begin{gathered}
U(a, t)=0, \quad U(b, t)=0, \\
U_{x}(a, t)=U_{x}(b, t)=0, \\
U_{x x}(a, t)=U_{x x}(b, t)=0, \\
U_{x x x}(a, t)=U_{x x x}(b, t)=0, \quad t>0,
\end{gathered}
$$

and the initial condition

$$
U(x, 0)=f(x) \quad a \leq x \leq b .
$$

In order to be able to apply the numerical method, the solution region of the problem is restricted over an interval $a \leq x \leq b$. Space interval $[a, b]$ is partitioned into uniformly sized finite elements of length $h$ by the nodes $x_{m}$ such that $a=x_{0}<x_{1}<\cdots<x_{N-1}<x_{N}=b$ and $h=x_{m+1}-x_{m}, m=$ $-3,-2, \ldots, N+2, N+3$. The set of septic B-spline functions $\left\{\phi_{-3}(x), \phi_{-2}(x), \phi_{-1}(x), \ldots, \phi_{N+1}(x), \phi_{N+2}(x), \phi_{N+3}(x)\right\}$ forms a basis over the problem domain $[a, b]$. A global approximation $U_{N}(x, t)$ is expressed in terms of septic Bsplines as

$$
U_{N}(x, t)=\sum_{i=-3}^{N+3} \phi_{i}(x) \delta_{i}(t)
$$

where $\delta_{i}(t)$ s are time dependent parameters to be determined from the initial, boundary, and collocation conditions.
Septic B-splines $\phi_{m}(x),(m=-3(1) N+3)$, at the knots $x_{m}$ are defined over the interval $[a, b]$ by $[40]$

$$
\phi_{m}(x)=\frac{1}{h^{7}}\left\{\begin{array}{cc}
\left(x-x_{m-4}\right)^{7} & {\left[x_{m-4}, x_{m-3}\right]} \\
\left(x-x_{m-4}\right)^{7}-8\left(x-x_{m-3}\right)^{7} & {\left[x_{m-3}, x_{m-2}\right]} \\
\left(x-x_{m-4}\right)^{7}-8\left(x-x_{m-3}\right)^{7} \\
+28\left(x-x_{m-2}\right)^{7} \\
{\left[x_{m-2}, x_{m-1}\right]} \\
\left(x-x_{m-4}\right)^{7}-8\left(x-x_{m-3}\right)^{7} \\
+28\left(x-x_{m-2}\right)^{7}-56\left(x-x_{m-1}\right)^{7}-8\left(x_{m+3}-x\right)^{7}-56\left(x_{m+1}-x\right)^{7} \\
{\left[x_{m-1}, x_{m}\right]} \\
\left(x_{m+4}-x\right)^{7}-8\left(x_{m+3}-x\right)^{7} \\
+28\left(x_{m+2}-x\right)^{7} \\
{\left[x_{m+1}, x_{m+2}\right]} \\
\left(x_{m+4}-x\right)^{7}-8\left(x_{m+3}-x\right)^{7} \\
\left(x_{m+4}-x\right)^{7} \\
{\left[x_{m+2}, x_{m+3}\right]} \\
0 \\
\text { otherwise. }
\end{array}\right.
$$

Using expansion (6) and trial function (7), the nodal values $\left(U_{N}\right)_{m}$ and their first, second, and third derivatives $\left(U_{N}\right)_{x},\left(U_{N}\right)_{x x},\left(U_{N}\right)_{x x x}$ can be calculated at the nodal points $x_{m}$ in terms of nodal parameters $\delta_{m}$ by the following set of equations:

$$
\begin{gathered}
\left(U_{N}\right)_{m}=\delta_{m-3}+120 \delta_{m-2}+1191 \delta_{m-1}+2416 \delta_{m} \\
+1191 \delta_{m+1}+120 \delta_{m+2}+\delta_{m+3}, \\
\left(U_{N x}\right)_{m}=\frac{7}{h}\left(-\delta_{m-3}-56 \delta_{m-2}-245 \delta_{m-1}\right. \\
\left.\quad+245 \delta_{m+1}+56 \delta_{m+2}+\delta_{m+3}\right), \\
\left(U_{N x x}\right)_{m}=\frac{42}{h^{2}}\left(\delta_{m-3}+24 \delta_{m-2}+15 \delta_{m-1}-80 \delta_{m}\right. \\
\left.\quad+15 \delta_{m+1}+24 \delta_{m+2}+\delta_{m+3}\right), \\
\left(U_{N x x x}\right)_{m}=\frac{210}{h^{3}}\left(-\delta_{m-3}-8 \delta_{m-2}+19 \delta_{m-1}\right. \\
\left.-19 \delta_{m+1}+8 \delta_{m+2}+\delta_{m+3}\right) .
\end{gathered}
$$

The splines $\phi_{m}(x)$ and their six principle derivatives vanish outside the interval $\left[x_{m-4}, x_{m+4}\right]$.

To apply the proposed method, Crank-Nicolson approximation for the space derivatives $U_{x}$ and $U_{x x}$ and usual first 
TABLE 1: Invariants and error norms for single solitary wave with $c=1, h=0.2, k=0.025,0 \leq x \leq 100$.

\begin{tabular}{lccccc}
\hline$t$ & $I_{1}$ & $I_{2}$ & $I_{3}$ & $L_{2} \times 10^{3}$ & $L_{\infty} \times 10^{3}$ \\
\hline 0 & 4.4428661 & 3.2998227 & 1.4142046 & 0.00000000 & 0.00000000 \\
1 & 4.4428661 & 3.2998085 & 1.4142188 & 0.28537793 & 0.16594258 \\
2 & 4.4428661 & 3.2997808 & 1.4142465 & 0.56248008 & 0.31854916 \\
3 & 4.4428661 & 3.2997573 & 1.4142700 & 0.82836630 & 0.45535369 \\
4 & 4.4428661 & 3.2997415 & 1.4142858 & 1.08566992 & 0.58528925 \\
5 & 4.44286601 & 3.2997313 & 1.4142960 & 1.33772774 & 0.71261445 \\
6 & 4.4428661 & 3.2997248 & 1.4143025 & 1.58675627 & 0.83879372 \\
7 & 4.4428661 & 3.2997207 & 1.4143067 & 1.83403948 & 0.96441682 \\
8 & 4.4428661 & 3.2997180 & 1.4143093 & 2.08032250 & 1.08975930 \\
9 & 4.4428661 & 3.2997162 & 1.4143111 & 2.32602024 & 1.21494581 \\
10 & 4.4428661 & 3.2997151 & 1.4143122 & 2.57148152 & 1.34021078 \\
\hline
\end{tabular}

TABLE 2: Errors and invariants for single solitary wave with $c=1, h=0.2, k=0.025,0 \leq x \leq 100$, at $t=10$.

\begin{tabular}{|c|c|c|c|c|c|}
\hline Method & $I_{1}$ & $I_{2}$ & $I_{3}$ & $L_{2} \times 10^{3}$ & $L_{\infty} \times 10^{3}$ \\
\hline Analytical & 4.4428829 & 3.2998316 & 1.4142135 & 0 & 0 \\
\hline Present & 4.4428661 & 3.2997151 & 1.4143122 & 2.57148 & 1.334021 \\
\hline$[6]$ & 4.44288 & 3.29981 & 1.41416 & 3.00533 & 1.68749 \\
\hline Cubic B-splines coll-CN [29] & 4.442 & 3.299 & 1.413 & 16.39 & 9.24 \\
\hline Cubic B-splines coll-PA-CN+ [29] & 4.440 & 3.296 & 1.411 & 20.3 & 11.2 \\
\hline Cubic B-splines coll [30] & 4.44288 & 3.29983 & 1.41420 & 9.30196 & 5.43718 \\
\hline MQ [35] & 4.4428829 & 3.29978 & 1.414163 & 3.914 & 2.019 \\
\hline IMQ [35] & 4.4428611 & 3.29978 & 1.414163 & 3.914 & 2.019 \\
\hline IQ [35] & 4.4428794 & 3.29978 & 1.414163 & 3.914 & 2.019 \\
\hline GA [35] & 4.4428829 & 3.29978 & 1.414163 & 3.914 & 2.019 \\
\hline TPS [35] & 4.4428821 & 3.29972 & 1.414104 & 4.428 & 2.306 \\
\hline Quintic B-splines coll [36] & 4.4428661 & 3.2997108 & 1.4143165 & 2.58891 & 1.35164 \\
\hline
\end{tabular}

TABLE 3: Invariants and error norms for single solitary wave with $c=0.3, h=0.1, k=0.01,0 \leq x \leq 100$.

\begin{tabular}{|c|c|c|c|c|c|}
\hline$t$ & $I_{1}$ & $I_{2}$ & $I_{3}$ & $L_{2} \times 10^{4}$ & $L_{\infty} \times 10^{4}$ \\
\hline 0 & 3.5819531 & 1.3450721 & 0.1537217 & 0.0000000 & 0.0000000 \\
\hline 2 & 3.5819531 & 1.3450719 & 0.1537219 & 0.0373696 & 0.0211791 \\
\hline 4 & 3.5819531 & 1.3450715 & 0.1537223 & 0.0711480 & 0.0387624 \\
\hline 6 & 3.5819531 & 1.3450711 & 0.1537227 & 0.1001141 & 0.0515117 \\
\hline 8 & 3.5819531 & 1.3450708 & 0.1537231 & 0.1249329 & 0.0614203 \\
\hline 10 & 3.5819531 & 1.3450705 & 0.1537234 & 0.1466243 & 0.0700260 \\
\hline 12 & 3.5819531 & 1.3450702 & 0.1537236 & 0.1659668 & 0.0775889 \\
\hline 14 & 3.5819531 & 1.3450700 & 0.1537238 & 0.1833628 & 0.0844911 \\
\hline 16 & 3.5819531 & 1.3450698 & 0.1537240 & 0.2015361 & 0.0909663 \\
\hline 18 & 3.5819531 & 1.3450697 & 0.1537241 & 0.2560750 & 0.0993420 \\
\hline 20 & 3.5819531 & 1.3450696 & 0.1537243 & 0.3585031 & 0.1702101 \\
\hline $20[6]$ & 3.58197 & 1.34508 & 0.153723 & 0.645295 & 0.301923 \\
\hline $20[30]$ & 3.58197 & 1.34508 & 0.153723 & 6.06885 & 2.96650 \\
\hline $20[34]$ & 3.581967 & 1.345076 & 0.153723 & 0.508927 & 0.222284 \\
\hline 20 [35] MQ & 3.5819665 & 1.3450764 & 0.153723 & 0.51498 & 0.22551 \\
\hline 20 [35] IMQ & 3.5819664 & 1.3450764 & 0.153723 & 0.51498 & 0.22551 \\
\hline 20 [35] IQ & 3.5819654 & 1.3450764 & 0.153723 & 0.51498 & 0.22551 \\
\hline 20 [35] GA & 3.5819665 & 1.3450764 & 0.153723 & 0.51498 & 0.22551 \\
\hline 20 [35] TPS & 3.5819663 & 1.3450759 & 0.153723 & 0.51498 & 0.26605 \\
\hline $20[36]$ & 3.5820204 & 1.3450974 & 0.1537250 & 0.8112594 & 0.3569076 \\
\hline
\end{tabular}


TABLE 4: Invariants and error norms for single solitary wave with $c=0.6, h=0.1, k=0.1,-40 \leq x \leq 60$.

\begin{tabular}{lcccccc}
\hline$t$ & $I_{1}$ & $I_{2}$ & $I_{3}$ & $L_{2} \times 10^{4}$ & $L_{\infty} \times 10^{4}$ & CPU Time \\
\hline 4 & 8.070902 & 4.100549 & 14.361115 & 2.716636 & 1.323585 & $0.437 \mathrm{~s}$ \\
8 & 8.070925 & 4.100534 & 14.361111 & 5.240855 & 2.458097 & $0.908 \mathrm{~s}$ \\
12 & 8.070943 & 4.100519 & 14.361103 & 7.543043 & 3.372256 & $1.435 \mathrm{~s}$ \\
16 & 8.070949 & 4.100503 & 14.361089 & 9.662561 & 4.154427 & $1.774 \mathrm{~s}$ \\
20 & 8.070921 & 4.100489 & 14.361074 & 11.660256 & 4.862191 \\
\hline
\end{tabular}

TABLE 5: Invariants and error norms for single solitary wave with $c=0.18, h=0.1, k=0.1,-80 \leq x \leq 120$.

\begin{tabular}{lcccccc}
\hline$t$ & $I_{1}$ & $I_{2}$ & $I_{3}$ & $L_{2} \times 10^{5}$ & $L_{\infty} \times 10^{5}$ & CPU Time \\
\hline 4 & 7.809873 & 2.129887 & 7.031111 & 3.077451 & 1.138290 & $0.801 \mathrm{~s}$ \\
8 & 7.809875 & 2.129887 & 7.031112 & 6.124574 & 2.313797 & 3.5755915 \\
12 & 7.809877 & 2.129887 & 7.031112 & 9.106266 & $2.470 \mathrm{~s}$ \\
16 & 7.809879 & 2.129887 & 7.031113 & 11.967534 & 4.530312 & $3.124 \mathrm{~s}$ \\
20 & 7.809880 & 2.129887 & 7.031115 & 14.731556 & 5.538799 \\
\hline
\end{tabular}

order forward difference formula for the time derivative of the $U$ in (3) have been used, which lead to

$$
\begin{aligned}
& \frac{U^{n+1}-U^{n}}{\Delta t}+\frac{U_{x}^{n+1}+U_{x}^{n}}{2}+6 \frac{\left(U^{2} U_{x}\right)^{n+1}+\left(U^{2} U_{x}\right)^{n}}{2} \\
& -\mu \frac{U_{x x}^{n+1}-U_{x x}^{n}}{\Delta t}=0 .
\end{aligned}
$$

In order to linearize the nonlinear term $\left(U^{2} U_{x}\right)^{n+1}$, we can write the term as follows,

$$
U^{2} U_{x}=U U U_{x}
$$

and apply the linearization form introduced by Rubin and Graves [37]

$$
\left(U U_{x}\right)^{n+1}=U^{n+1} U_{x}^{n}+U^{n} U_{x}^{n+1}-U^{n} U_{x}^{n}
$$

to (9), and we get

$$
\begin{aligned}
U^{n+1} & +\frac{\Delta t}{2} U_{x}^{n+1}+3 \Delta t U^{n+1} \\
& \times\left(U^{n+1} U_{x}^{n}+U^{n} U_{x}^{n+1}-U^{n} U_{x}^{n}\right)-\mu U_{x x}^{n+1} \\
= & U^{n}-\frac{\Delta t}{2} U_{x}^{n}-3 \Delta t U^{n}\left(U U_{x}\right)^{n}-\mu U_{x x}^{n} .
\end{aligned}
$$

Substituting the approximate solution $U_{N}$ and putting the nodal values of $U$ and its derivatives given by (8) into (12) one obtains the following iterative system for $m=0,1, \ldots, N$ :

$$
\begin{aligned}
& \delta_{m-3}^{n+1}\left(1+\alpha_{1}-\alpha_{2}-\alpha_{3}\right)+\delta_{m-2}^{n+1}\left(120+120 \alpha_{1}-56 \alpha_{2}-24 \alpha_{3}\right) \\
& \quad+\delta_{m-1}^{n+1}\left(1191+1191 \alpha_{1}-245 \alpha_{2}-15 \alpha_{3}\right) \\
& \quad+\delta_{m}^{n+1}\left(2416+2416 \alpha_{1}+80 \alpha_{3}\right)
\end{aligned}
$$

$$
\begin{aligned}
& +\delta_{m+1}^{n+1}\left(1191+1191 \alpha_{1}+245 \alpha_{2}-15 \alpha_{3}\right) \\
& +\delta_{m+2}^{n+1}\left(120+120 \alpha_{1}+56 \alpha_{2}-24 \alpha_{3}\right) \\
& +\delta_{m+3}^{n+1}\left(1+\alpha_{1}+\alpha_{2}-\alpha_{3}\right) \\
& =\delta_{m-3}^{n}\left(1-\alpha_{1}+\alpha_{2}-\alpha_{3}\right) \\
& +\delta_{m-2}^{n}\left(120-120 \alpha_{1}+56 \alpha_{2}-24 \alpha_{3}\right) \\
& +\delta_{m-1}^{n}\left(1191-1191 \alpha_{1}+245 \alpha_{2}-15 \alpha_{3}\right) \\
& +\delta_{m}^{n}\left(2416-2416 \alpha_{1}+80 \alpha_{3}\right) \\
& +\delta_{m+1}^{n}\left(1191-1191 \alpha_{1}-245 \alpha_{2}-15 \alpha_{3}\right) \\
& +\delta_{m+2}^{n}\left(120-120 \alpha_{1}-56 \alpha_{2}-24 \alpha_{3}\right) \\
& +\delta_{m+3}^{n}\left(1-\alpha_{1}-\alpha_{2}-\alpha_{3}\right),
\end{aligned}
$$

where

$$
\alpha_{1}=3 \Delta t, \quad \alpha_{2}=\frac{7 \Delta t}{2 h}, \quad \alpha_{3}=\frac{42 \mu}{h^{2}}
$$

The newly obtained iterative system (13) consists of $N+1$ linear equation in $N+7$ unknowns $\left(\delta_{-3}, \delta_{-2}, \delta_{-1}, \delta_{0}, \ldots\right.$, $\left.\delta_{N}, \delta_{N+1}, \delta_{N+2}, \delta_{N+3}\right)^{T}$. To obtain a unique solution of this system, six additional constraints are required. Applying the boundary conditions (4) and using the values of (8), these constraints are used and this enables us to eliminate the unknowns $\delta_{-3}, \delta_{-2}, \delta_{-1}, \delta_{0}, \ldots, \delta_{N}, \delta_{N+1}, \delta_{N+2}, \delta_{N+3}$ from system (13). So system (13) is reduced to a septa-diagonal system of $(N+1)$ linear equations in $(N+1)$ unknowns given by $A \delta^{n+1}=B \delta^{n}$, where $\delta^{n}=\left(\delta_{0}, \delta_{1}, \delta_{2}, \ldots, \delta_{N-2}, \delta_{N-1}, \delta_{N}\right)^{T}$. The coefficient matrixes are given by 
TABLE 6: Comparison of the invariants for the interaction of two solitary waves with results from [30] with $h=0.2, k=0.025$ in the region $0 \leq x \leq 250$.

\begin{tabular}{|c|c|c|c|c|c|c|c|}
\hline \multirow[b]{2}{*}{$t$} & \multicolumn{3}{|c|}{ Present method } & \multirow[b]{2}{*}{ CPU Time } & \multicolumn{3}{|c|}{ [30] } \\
\hline & $I_{1}$ & $I_{2}$ & $I_{3}$ & & $I_{1}$ & $I_{2}$ & $I_{3}$ \\
\hline 0 & 11.4676 & 14.6292 & 22.8803 & - & 11.4677 & 14.6291 & 22.8806 \\
\hline 2 & 11.4678 & 14.6282 & 22.8812 & $0.209 \mathrm{~s}$ & 11.4677 & 14.6292 & 22.8807 \\
\hline 4 & 11.4679 & 14.6282 & 22.8812 & $0.413 \mathrm{~s}$ & 11.4677 & 14.6292 & 22.8807 \\
\hline 6 & 11.4681 & 14.6181 & 22.8914 & $1.101 \mathrm{~s}$ & 11.4677 & 14.6295 & 22.8806 \\
\hline 8 & 11.4675 & 14.1393 & 23.3702 & $1.219 \mathrm{~s}$ & 11.4677 & 14.6451 & 22.8454 \\
\hline 10 & 11.4674 & 14.0502 & 23.4592 & $1.421 \mathrm{~s}$ & 11.4677 & 14.5963 & 22.8913 \\
\hline 12 & 11.4685 & 14.6817 & 22.8278 & $2.027 \mathrm{~s}$ & 11.4677 & 14.6287 & 22.8814 \\
\hline 14 & 11.4687 & 14.6649 & 22.8446 & $2.228 \mathrm{~s}$ & 11.4677 & 14.6295 & 22.8807 \\
\hline 16 & 11.4688 & 14.6459 & 22.8635 & $2.432 \mathrm{~s}$ & 11.4677 & 14.6294 & 22.8808 \\
\hline 18 & 11.4690 & 14.6370 & 22.8725 & $3.004 \mathrm{~s}$ & 11.4677 & 14.6293 & 22.8809 \\
\hline 20 & 11.4691 & 14.6331 & 22.8763 & $3.239 \mathrm{~s}$ & 11.4677 & 14.6292 & 22.8809 \\
\hline $20[6]$ & 11.4677 & 14.6299 & 22.8806 & & & & \\
\hline $20[34]$ & 11.4677 & 14.5830 & 22.6965 & & & & \\
\hline 20 [35] MQ & 11.4676 & 14.5830 & 22.6965 & & & & \\
\hline 20 [35] IMQ & 11.4676 & 14.5830 & 22.6965 & & & & \\
\hline 20 [35] IQ & 11.4676 & 14.5830 & 22.6965 & & & & \\
\hline 20 [35] GA & 11.4676 & 14.5830 & 22.6965 & & & & \\
\hline 20 [35] TPS & 11.4677 & 14.5824 & 22.6942 & & & & \\
\hline $20[36]$ & 11.4691 & 14.6331 & 22.8764 & & & & \\
\hline
\end{tabular}

TABLE 7: Comparison of invariants for the interaction of three solitary waves with results from [30] with $h=0.2, k=0.025$ in the region $0 \leq x \leq 250$.

\begin{tabular}{|c|c|c|c|c|c|c|c|}
\hline \multirow[b]{2}{*}{$t$} & \multicolumn{3}{|c|}{ Present method } & \multirow[b]{2}{*}{ CPU Time } & \multicolumn{3}{|c|}{$[30]$} \\
\hline & $I_{1}$ & $I_{2}$ & $I_{3}$ & & $I_{1}$ & $I_{2}$ & $I_{3}$ \\
\hline 0 & 14.9800 & 15.8374 & 23.0081 & - & 13.6891 & 15.4549 & 22.8816 \\
\hline 5 & 14.9804 & 15.7991 & 23.0465 & $0.536 \mathrm{~s}$ & 13.6891 & 15.3109 & 22.6939 \\
\hline 10 & 14.9783 & 14.3275 & 24.5180 & $1.442 \mathrm{~s}$ & 13.6891 & 15.6514 & 22.8388 \\
\hline 15 & 14.9809 & 15.5048 & 23.3408 & $2.349 \mathrm{~s}$ & 13.6891 & 15.6548 & 22.9347 \\
\hline 20 & 14.9814 & 15.7504 & 23.0952 & $3.251 \mathrm{~s}$ & 13.6891 & 15.6557 & 22.9330 \\
\hline 25 & 14.9819 & 15.8496 & 22.9960 & $4.162 \mathrm{~s}$ & 13.6892 & 15.6559 & 22.9336 \\
\hline 30 & 14.9823 & 15.8626 & 22.9829 & $5.063 \mathrm{~s}$ & 13.6894 & 15.6559 & 22.9348 \\
\hline 35 & 14.9828 & 15.8622 & 22.9833 & $5.573 \mathrm{~s}$ & 13.6913 & 15.6564 & 22.9343 \\
\hline 40 & 14.9832 & 15.8605 & 22.9851 & $6.481 \mathrm{~s}$ & 13.7015 & 15.6566 & 22.9335 \\
\hline 45 & 14.9461 & 15.8613 & 22.9843 & $7.388 \mathrm{~s}$ & 13.7043 & 15.6563 & 22.9303 \\
\hline $45[34]$ & 13.7043 & 15.6563 & 22.9303 & & & & \\
\hline 45 [35] MQ & 14.9681 & 15.7343 & 22.5966 & & & & \\
\hline 45 [35] IMQ & 14.9680 & 15.7343 & 22.5966 & & & & \\
\hline 45 [35] IQ & 14.9681 & 15.7343 & 22.5966 & & & & \\
\hline 45 [35] GA & 14.9681 & 15.7343 & 22.5966 & & & & \\
\hline 45 [35] TPS & 14.9682 & 15.7337 & 22.5944 & & & & \\
\hline $45[36]$ & 14.7145 & 15.4927 & 23.3529 & & & & \\
\hline
\end{tabular}




$$
\begin{aligned}
& A=\left[\begin{array}{cccccccccc}
a_{11} & a_{12} & a_{13} & a_{14} & & & & & & \\
a_{21} & a_{22} & a_{23} & a_{24} & a_{25} & & & & & \\
a_{31} & a_{32} & a_{33} & a_{34} & a_{35} & a_{36} & & & & \\
& & & & \ddots & & & & & \\
& & a_{m, m-3} & a_{m, m-2} & a_{m, m-1} & a_{m, m} & a_{m, m+1} & a_{m, m+2} & a_{m, m+3} & \\
& & & & & \ddots & & & & \\
& & & & a_{n-1, n-4} & a_{n-1, n-3} & a_{n-1, n-2} & a_{n-1, n-1} & a_{n-1, n} & a_{n-1, n+1} \\
& & & & & a_{n, n-3} & a_{n, n-2} & a_{n, n-1} & a_{n, n} & a_{n, n+1} \\
& & & & & & a_{n+1, n-2} & a_{n+1, n-1} & a_{n+1, n} & a_{n+1, n+1}
\end{array}\right], \\
& B=\left[\begin{array}{cccccccccc}
b_{11} & b_{12} & b_{13} & b_{14} & & & & & & \\
b_{21} & b_{22} & b_{23} & b_{24} & b_{25} & & & & & \\
b_{31} & b_{32} & b_{33} & b_{34} & b_{35} & b_{36} & & & & \\
& & & & \ddots & & & & & \\
& & b_{m, m-3} & b_{m, m-2} & b_{m, m-1} & b_{m, m} & b_{m, m+1} & b_{m, m+2} & b_{m, m+3} & \\
& & & & & \ddots & & & & \\
& & & & b_{n-1, n-4} & b_{n-1, n-3} & b_{n-1, n-2} & b_{n-1, n-1} & b_{n-1, n} & b_{n-1, n+1} \\
& & & & & b_{n, n-3} & b_{n, n-2} & b_{n, n-1} & b_{n, n} & b_{n, n+1} \\
& & & & & & b_{n+1, n-2} & b_{n+1, n-1} & b_{n+1, n} & b_{n+1, n+1}
\end{array}\right],
\end{aligned}
$$

where

$$
\begin{aligned}
& a_{11}=-\frac{41472}{297} \alpha_{3}, \quad b_{11}=-\frac{41472}{297} \alpha_{3}, \\
& a_{12}=-\frac{73224}{297} \alpha_{3}, \quad b_{12}=-\frac{73224}{297} \alpha_{3}, \\
& a_{13}=-\frac{20736}{297} \alpha_{3}, \quad b_{13}=-\frac{20736}{297} \alpha_{3}, \\
& a_{14}=-\frac{648}{297} \alpha_{3}, \quad b_{14}=-\frac{648}{297} \alpha_{3}, \\
& a_{21}=\frac{215411}{297}+\frac{215411}{297} \alpha_{1}-\frac{11761}{297} \alpha_{2}+\frac{17893}{297} \alpha_{3}, \\
& b_{21}=\frac{215411}{297}-\frac{215411}{297} \alpha_{1}+\frac{11761}{297} \alpha_{2}+\frac{17893}{297} \alpha_{3} \text {, } \\
& a_{22}=\frac{616822.5}{297}+\frac{616822.5}{297} \alpha_{1}+\frac{43513.5}{297} \alpha_{2}+\frac{38665.5}{297} \alpha_{3} \\
& b_{22}=\frac{616822.5}{297}-\frac{616822.5}{297} \alpha_{1}-\frac{43513.5}{297} \alpha_{2}+\frac{38665.5}{297} \alpha_{3} \text {, } \\
& a_{23}=\frac{340284}{297}+\frac{340284}{297} \alpha_{1}+\frac{78528}{297} \alpha_{2}-\frac{2532}{297} \alpha_{3}, \\
& b_{23}=\frac{340284}{297}-\frac{340284}{297} \alpha_{1}-\frac{78528}{297} \alpha_{2}-\frac{2532}{297} \alpha_{3}, \\
& a_{24}=\frac{35525.5}{297}+\frac{35525.5}{297} \alpha_{1}+\frac{16682.5}{297} \alpha_{2}-\frac{7109.5}{297} \alpha_{3}, \\
& b_{24}=\frac{35525.5}{297}+\frac{35525.5}{297} \alpha_{1}+\frac{16682.5}{297} \alpha_{2}-\frac{7109.5}{297} \alpha_{3} \text {, }
\end{aligned}
$$

$$
\begin{aligned}
& a_{25}=1+\alpha_{1}+\alpha_{2}-\alpha_{3}, \quad b_{25}=1-\alpha_{1}-\alpha_{2}-\alpha_{3}, \\
& a_{31}=\frac{34432}{297}+\frac{34432}{297} \alpha_{1}-\frac{15424}{297} \alpha_{2}-\frac{5920}{297} \alpha_{3}, \\
& b_{31}=\frac{34432}{297}-\frac{34432}{297} \alpha_{1}+\frac{15424}{297} \alpha_{2}-\frac{5920}{297} \alpha_{3}, \\
& a_{32}=\frac{352833}{297}+\frac{352833}{297} \alpha_{1}-\frac{71871}{297} \alpha_{2}-\frac{3561}{297} \alpha_{3}, \\
& b_{32}=\frac{352833}{297}-\frac{352833}{297} \alpha_{1}+\frac{71871}{297} \alpha_{2}-\frac{3561}{297} \alpha_{3}, \\
& a_{33}=\frac{717432}{297}+\frac{717432}{297} \alpha_{1}+\frac{120}{297} \alpha_{2}+\frac{23880}{297} \alpha_{3}, \\
& b_{33}=\frac{717432}{297}-\frac{717432}{297} \alpha_{1}-\frac{120}{297} \alpha_{2}+\frac{23880}{297} \alpha_{3}, \\
& b_{35}=120-120 \alpha_{1}-56 \alpha_{2}-24 \alpha_{3}, \\
& b_{m, m-3}=1+\alpha_{1}-\alpha_{2}-\alpha_{3}, \\
& a_{34}=\frac{353726}{297}+\frac{353726}{297} \alpha_{1}+\frac{72766}{297} \alpha_{2}-\frac{4454}{297} \alpha_{3}, \\
& b_{34}=\frac{353726}{297}-\frac{353726}{297} \alpha_{1}-\frac{72766}{297} \alpha_{2}-\frac{4454}{297} \alpha_{3}, \\
& a_{36}, \alpha_{2}, \alpha_{3}, \\
& b_{36}, \alpha_{2}+56 \alpha_{2}-24 \alpha_{3},
\end{aligned}
$$


$a_{m, m-2}=120+120 \alpha_{1}-56 \alpha_{2}-24 \alpha_{3}$,

$b_{m, m-2}=120-120 \alpha_{1}+56 \alpha_{2}-24 \alpha_{3}$,

$a_{m, m-1}=1191+1191 \alpha_{1}-245 \alpha_{2}-15 \alpha_{3}$,

$b_{m, m-1}=1191-1191 \alpha_{1}+245 \alpha_{2}-15 \alpha_{3}$,

$a_{m, m}=2416+2416 \alpha_{1}+80 \alpha_{3}$,

$b_{m, m}=2416-2416 \alpha_{1}+80 \alpha_{3}$,

$a_{m, m+1}=1191+1191 \alpha_{1}+245 \alpha_{2}-15 \alpha_{3}$,

$b_{m, m+1}=1191-1191 \alpha_{1}-245 \alpha_{2}-15 \alpha_{3}$,

$a_{m, m+2}=120+120 \alpha_{1}+56 \alpha_{2}-24 \alpha_{3}$,

$b_{m, m+2}=120-120 \alpha_{1}-56 \alpha_{2}-24 \alpha_{3}$,

$a_{m, m+3}=1+\alpha_{1}+\alpha_{2}-\alpha_{3}$

$b_{m, m+3}=1-\alpha_{1}-\alpha_{2}-\alpha_{3}$,

$m=4(1) n-2$,

$a_{n-1, n-4}=1+\alpha_{1}-\alpha_{2}-\alpha_{3}$,

$b_{n-1, n-4}=1-\alpha_{1}+\alpha_{2}-\alpha_{3}$,

$a_{n-1, n-3}=120+120 \alpha_{1}-56 \alpha_{2}-24 \alpha_{3}$,

$b_{n-1, n-3}=120-120 \alpha_{1}+56 \alpha_{2}-24 \alpha_{3}$,

$a_{n-1, n-2}=\frac{353726}{297}+\frac{353726}{297} \alpha_{1}-\frac{72766}{297} \alpha_{2}-\frac{4454}{297} \alpha_{3}$,

$b_{n-1, n-2}=\frac{353726}{297}-\frac{353726}{297} \alpha_{1}+\frac{72766}{297} \alpha_{2}-\frac{4454}{297} \alpha_{3}$,

$a_{n-1, n-1}=\frac{717432}{297}+\frac{717432}{297} \alpha_{1}-\frac{120}{297} \alpha_{2}+\frac{23880}{297} \alpha_{3}$,

$b_{n-1, n-1}=\frac{717432}{297}-\frac{717432}{297} \alpha_{1}+\frac{120}{297} \alpha_{2}+\frac{23880}{297} \alpha_{3}$,

$a_{n-1, n}=\frac{352833}{297}+\frac{352833}{297} \alpha_{1}+\frac{71871}{297} \alpha_{2}-\frac{3561}{297} \alpha_{3}$,

$b_{n-1, n}=\frac{352833}{297}-\frac{352833}{297} \alpha_{1}-\frac{71871}{297} \alpha_{2}-\frac{3561}{297} \alpha_{3}$,

$a_{n-1, n+1}=\frac{34432}{297}+\frac{34432}{297} \alpha_{1}+\frac{15424}{297} \alpha_{2}-\frac{5920}{297} \alpha_{3}$,

$b_{n-1, n+1}=\frac{34432}{297}-\frac{34432}{297} \alpha_{1}-\frac{15424}{297} \alpha_{2}-\frac{5920}{297} \alpha_{3}$,

$a_{n, n-3}=1+\alpha_{1}-\alpha_{2}-\alpha_{3}, \quad b_{n, n-3}=1-\alpha_{1}+\alpha_{2}-\alpha_{3}$,

$$
\begin{aligned}
& a_{n, n-2}=\frac{35525.5}{297}+\frac{35525.5}{297} \alpha_{1}-\frac{16682.5}{297} \alpha_{2}-\frac{7109.5}{297} \alpha_{3} \text {, } \\
& b_{n, n-2}=\frac{35525.5}{297}-\frac{35525.5}{297} \alpha_{1}+\frac{16682.5}{297} \alpha_{2}-\frac{7109.5}{297} \alpha_{3} \text {, } \\
& a_{n, n-1}=\frac{340284}{297}+\frac{340284}{297} \alpha_{1}-\frac{78528}{297} \alpha_{2}-\frac{2532}{297} \alpha_{3} \text {, } \\
& b_{n, n-1}=\frac{340284}{297}-\frac{340284}{297} \alpha_{1}+\frac{78528}{297} \alpha_{2}-\frac{2532}{297} \alpha_{3}, \\
& a_{n, n}=\frac{616822.5}{297}+\frac{616822.5}{297} \alpha_{1}-\frac{43513.5}{297} \alpha_{2}+\frac{38665.5}{297} \alpha_{3} \text {, } \\
& b_{n, n}=\frac{616822.5}{297}-\frac{616822.5}{297} \alpha_{1}+\frac{43513.5}{297} \alpha_{2}+\frac{38665.5}{297} \alpha_{3} \text {, } \\
& a_{n, n+1}=\frac{215411}{297}+\frac{215411}{297} \alpha_{1}+\frac{11761}{297} \alpha_{2}+\frac{17893}{297} \alpha_{3}, \\
& b_{n, n+1}=\frac{215411}{297}-\frac{215411}{297} \alpha_{1}-\frac{11761}{297} \alpha_{2}+\frac{17893}{297} \alpha_{3}, \\
& a_{n+1, n-2}=-\frac{648}{297} \alpha_{3}, \quad b_{n+1, n-2}=-\frac{648}{297} \alpha_{3}, \\
& a_{n+1, n-1}=-\frac{20736}{297} \alpha_{3}, \quad b_{n+1, n-1}=-\frac{20736}{297} \alpha_{3}, \\
& a_{n+1, n}=-\frac{73224}{297} \alpha_{3}, \quad b_{n+1, n}=-\frac{73224}{297} \alpha_{3}, \\
& a_{n+1, n+1}=-\frac{41472}{297} \alpha_{3}, \quad b_{n+1, n+1}=-\frac{41472}{297} \alpha_{3} .
\end{aligned}
$$

Before starting the solution process, initial parameters $\delta^{0}$ must be determined by using the initial condition and the following derivatives at the boundaries:

$$
\begin{array}{ll}
U_{N}(x, 0)=U\left(x_{m}, 0\right) & m=0,1,2, \ldots, N, \\
\left(U_{N}\right)_{x}(a, 0)=0, & \left(U_{N}\right)_{x}(b, 0)=0, \\
\left(U_{N}\right)_{x x}(a, 0)=0, & \left(U_{N}\right)_{x x}(b, 0)=0, \\
\left(U_{N}\right)_{x x x}(a, 0)=0, & \left(U_{N}\right)_{x x x}(b, 0)=0 .
\end{array}
$$

So we have the following matrix form for the initial vector $\delta^{0}$ :

$$
M \delta^{0}=k,
$$


where

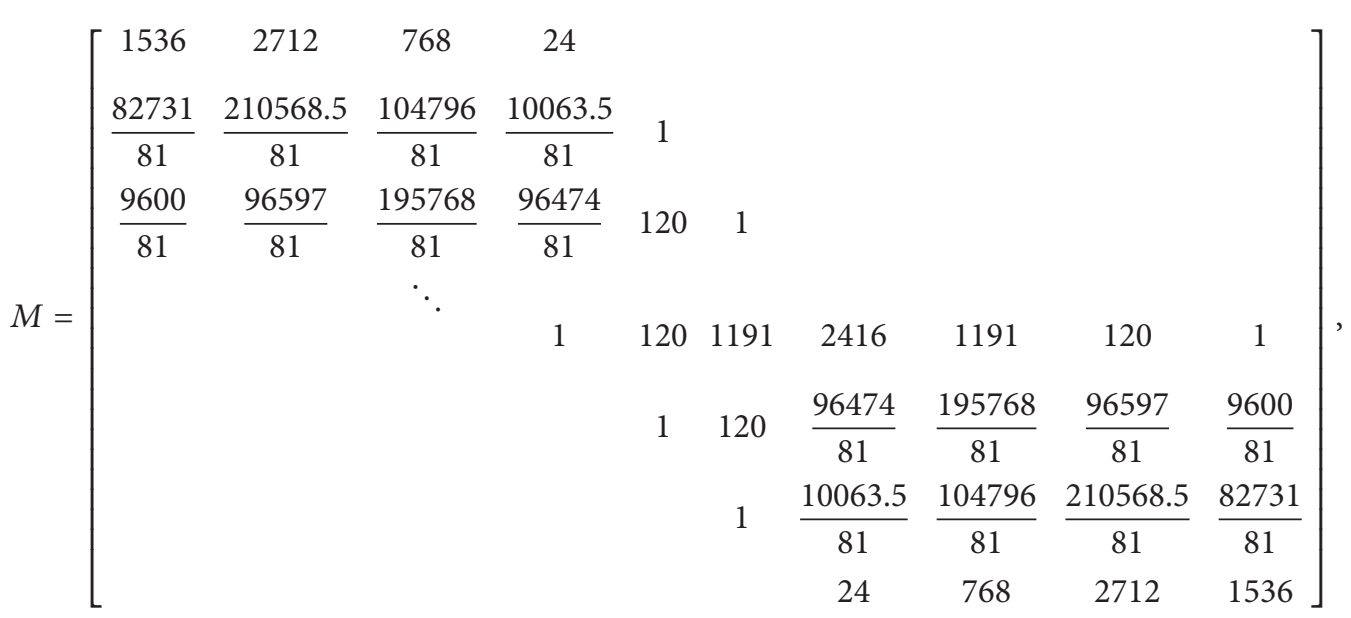

$\delta^{0}=\left(\delta_{0}, \delta_{1}, \delta_{2}, \ldots, \delta_{N-2}, \delta_{N-1}, \delta_{N}\right)^{T}$, and $k=\left(U\left(x_{0}, 0\right)\right.$, $\left.U\left(x_{1}, 0\right), \ldots, U\left(x_{N-1}, 0\right), U\left(x_{N}, 0\right)\right)^{T}$.

2.1. A Linear Stability Analysis. We have investigated stability analysis by applying the von-Neumann approach in which the growth factor of typical Fourier mode is given by

$$
\delta_{j}^{n}=\xi^{n} e^{i j k h}, \quad(i=\sqrt{-1}),
$$

where $k$ is a mode number and $h$ is the element size. To apply this method, we have linearized the nonlinear term $U^{2} U_{x}$ by considering $U^{2}$ as a constant such as $Z_{i}$ in (9). If we substitute (20) into the iterative system (13) we obtain the following equation:

$$
\xi^{n+1}=g \xi^{n}
$$

where $g$ is the growth factor. We have identified the collocation points with the nodes and used (8) to evaluate $U_{m}$ and its space derivatives in (3). This leads to a set of ordinary differential equations in the following form:

$$
\begin{aligned}
\dot{\delta}_{m-3}+ & 120 \dot{\delta}_{m-2}+1191 \dot{\delta}_{m-1}+2416 \dot{\delta}_{m}+1191 \dot{\delta}_{m+1} \\
+ & 120 \dot{\delta}_{m+2}+\dot{\delta}_{m+3}+\frac{7}{h}\left(1+6 Z_{m}\right) \\
\times & \left(-\delta_{m-3}-56 \delta_{m-2}-245 \delta_{m-1}\right. \\
& \left.+245 \delta_{m+1}+56 \delta_{m+2}+\delta_{m+3}\right) \\
- & \frac{42 \mu}{h^{2}}\left(\dot{\delta}_{m-3}+24 \dot{\delta}_{m-2}+15 \dot{\delta}_{m-1}-80 \dot{\delta}_{m}\right. \\
& \left.+15 \dot{\delta}_{m+1}+24 \dot{\delta}_{m+2}+\dot{\delta}_{m+3}\right)=0
\end{aligned}
$$

where $Z_{m}=\left(\delta_{m-3}+120 \delta_{m-2}+1191 \delta_{m-1}+2416 \delta_{m}+1191 \delta_{m+1}\right.$ $\left.+120 \delta_{m+2}+\delta_{m+3}\right)^{2}$. Here $\cdot$ denotes derivative with respect to time. If the parameters $\delta_{i}$ 's and their time derivatives in
(22) are discretized by the Crank-Nicolson formula and usual forward finite difference approximation, respectively,

$$
\delta_{i}=\frac{\delta^{n}+\delta^{n+1}}{2}, \quad \dot{\delta}_{i}=\frac{\delta^{n+1}-\delta^{n}}{\Delta t},
$$

we obtain a recurrence relationship between two time levels $n$ and $n+1$ relating two unknown parameters $\delta_{i}^{n+1}, \delta_{i}^{n}$ for $i=m-3, m-2, \ldots, m+2, m+3$,

$$
\begin{aligned}
\gamma_{1} \delta_{m-3}^{n+1} & +\gamma_{2} \delta_{m-2}^{n+1}+\gamma_{3} \delta_{m-1}^{n+1}+\gamma_{4} \delta_{m}^{n+1}+\gamma_{5} \delta_{m+1}^{n+1} \\
& +\gamma_{6} \delta_{m+2}^{n+1}+\gamma_{7} \delta_{m+3}^{n+1} \\
= & \gamma_{7} \delta_{m-3}^{n}+\gamma_{6} \delta_{m-2}^{n}+\gamma_{5} \delta_{m-1}^{n} \\
& +\gamma_{4} \delta_{m}^{n}+\gamma_{3} \delta_{m+1}^{n}+\gamma_{2} \delta_{m+2}^{n}+\gamma_{1} \delta_{m+3}^{n}
\end{aligned}
$$

where

$$
\begin{aligned}
& \gamma_{1}=1-E-M, \quad \gamma_{2}=120-56 E-24 M, \\
& \gamma_{3}=1191-245 E-15 M, \quad \gamma_{4}=2416+80 M, \\
& \gamma_{5}=1191+245 E-15 M, \quad \gamma_{6}=120+56 E-24 M, \\
& \gamma_{7}=1+E-M, \quad m=0,1, \ldots, N, \\
& E=\frac{7 \Delta t}{2 h}\left(1+6 Z_{m}\right), \quad M=\frac{42 \mu}{h^{2}} .
\end{aligned}
$$

Substituting the Fourier mode (20) into (24) leads to the growth factor of the form

$$
\xi=\frac{a-i b}{a+i b}
$$

where

$$
\begin{aligned}
& a=1208+40 M+(1191-15 M) \cos [h k] \\
& +(120-24 M) \cos [2 h k]+(1-M) \cos [3 h k], \\
& b=245 E \sin [h k]+56 E \sin [2 h k]+E \sin [3 h k] \text {, }
\end{aligned}
$$




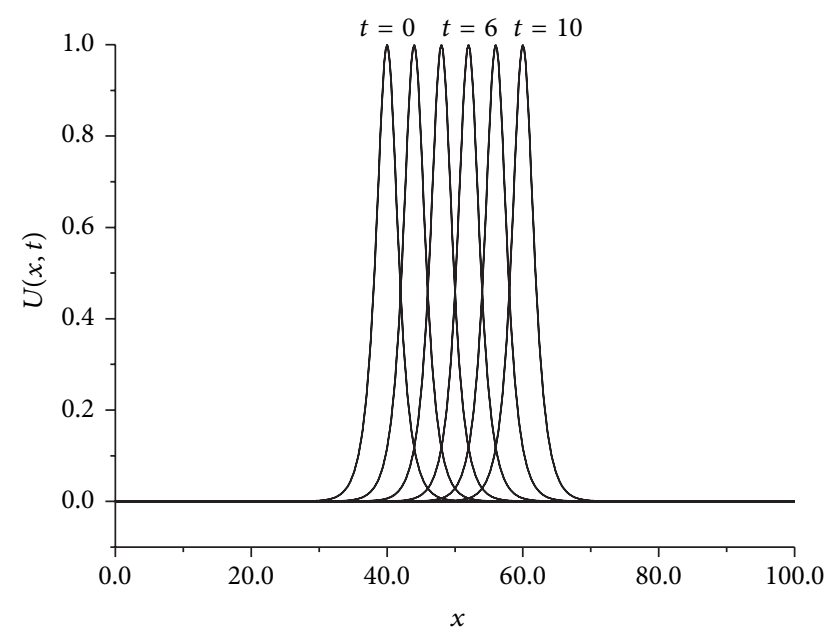

FIGURE 1: Single solitary wave with $c=1, h=0.2, \Delta t=0.025,0 \leq$ $x \leq 100$ at times $t=0,2,4,6,8$, and 10 .

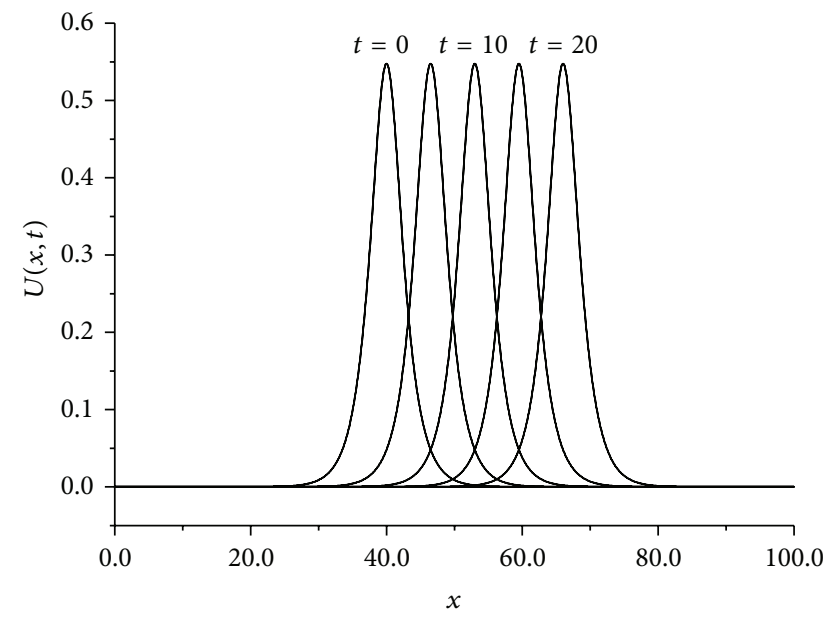

FIGURE 2: Single solitary wave with $c=0.3, h=0.1, \Delta t=0.01,0 \leq$ $x \leq 100$ at times $t=0,5,10,15$, and 20 .

$|\xi|^{2}=1$; therefore the linearized scheme is unconditionally stable.

\section{Numerical Examples and Results}

In this section, we have obtained numerical solution of the MRLW equation for motion of single solitary wave, interaction of two and three solitary waves, and development of the Maxwellian initial condition into solitary waves. Accuracy of the method is measured by using the following error norms:

$$
\begin{aligned}
& L_{2}=\left\|U^{\text {exact }}-U_{N}\right\|_{2} \simeq \sqrt{h \sum_{J=1}^{N}\left|U_{j}^{\text {exact }}-\left(U_{N}\right)_{j}\right|^{2}}, \\
& L_{\infty}=\left\|U^{\text {exact }}-U_{N}\right\|_{\infty} \simeq \max _{j}\left|U_{j}^{\text {exact }}-\left(U_{N}\right)_{j}\right| .
\end{aligned}
$$

The discrete conservation properties of the MRLW equation corresponding to mass, momentum, and energy are determined by finding the following three invariants [41]:

$$
\begin{aligned}
& I_{1}=\int_{a}^{b} U d x \simeq h \sum_{J=1}^{N} U_{j}^{n}, \\
& I_{2}=\int_{a}^{b}\left[U^{2}+\mu\left(U_{x}\right)^{2}\right] d x \simeq h \sum_{J=1}^{N}\left[\left(U_{j}^{n}\right)^{2}+\mu\left(U_{x}\right)_{j}^{n}\right], \\
& I_{3}=\int_{a}^{b}\left(U^{4}-\mu U_{x}^{2}\right) d x \simeq h \sum_{J=1}^{N}\left[\left(U_{j}^{n}\right)^{4}-\mu\left(U_{x}\right)_{j}^{n}\right] .
\end{aligned}
$$

3.1. The Motion of Single Solitary Wave. As a first problem, MRLW equation (3) is considered with the boundary conditions $U \rightarrow 0$ as $x \rightarrow \pm \infty$ and the initial condition

$$
U(x, 0)=\sqrt{c} \operatorname{sech}\left[p\left(x-x_{0}\right)\right] .
$$

Single solitary wave solution of the MRLW equation has an analytical solution of the form

$$
U(x, t)=\sqrt{c} \operatorname{sech}\left[p\left(x-(c+1) t-x_{0}\right)\right],
$$

where $p=\sqrt{c / \mu(c+1)}$ and $x_{0}$ and $c$ are arbitrary constants. This solution corresponds to motion of single solitary wave with amplitude $\sqrt{c}$, initially centered at $x_{0}$ and with wave velocity $1+\mu c$. For this problem the analytical values of the invariants are [29]

$$
I_{1}=\frac{\pi \sqrt{c}}{p}, \quad I_{2}=\frac{2 c}{p}+\frac{2 \mu p c}{3}, \quad I_{3}=\frac{4 c^{2}}{3 p}-\frac{2 \mu p c}{3} .
$$

For the computational work, two sets of parameters have been chosen and discussed. First of all, we have taken the parameters $c=1, \mu=1, h=0.2, x_{0}=40, k=0.025$ over the interval $[0,100]$ to compare our results with $[6$, $29,30,35,36]$. Thus, the solitary wave has an amplitude 1.0 and the computations are done up to time $t=10$ to obtain the invariants and error norms $L_{2}$ and $L_{\infty}$. Values of the three invariants and error norms are reported in Table 1 . It is clearly seen from the table that the error norms are satisfactorily small enough and the computed values of invariants are in good agreement with their analytical values $I_{1}=4.4428829, I_{2}=3.2998316$, and $I_{3}=1.4142135$. The percentage of the relative error of the conserved quantities $I_{1}, I_{2}$, and $I_{3}$ is calculated with respect to the conserved quantities at $t=0$. Percentage of relative changes of $I_{1}, I_{2}$, and $I_{3}$ is found to be $0.001 \times 10^{-3} \%, 3.261 \times 10^{-3} \%$, and $7.609 \times 10^{-3} \%$, respectively. Thus, the quantities $I_{1}, I_{2}$, and $I_{3}$ remain constant during the computer run. For this case in Table 2, we compare the values of the invariants and error norms obtained by using the present method and some earlier methods $[6,29,30,35,36]$. From the table, we observed that the error norms obtained by the present method are less than those of other methods $[6,29,30,35,36]$. Figure 1 shows the motion of solitary wave with $c=1, h=0.2, k=0.025$ 


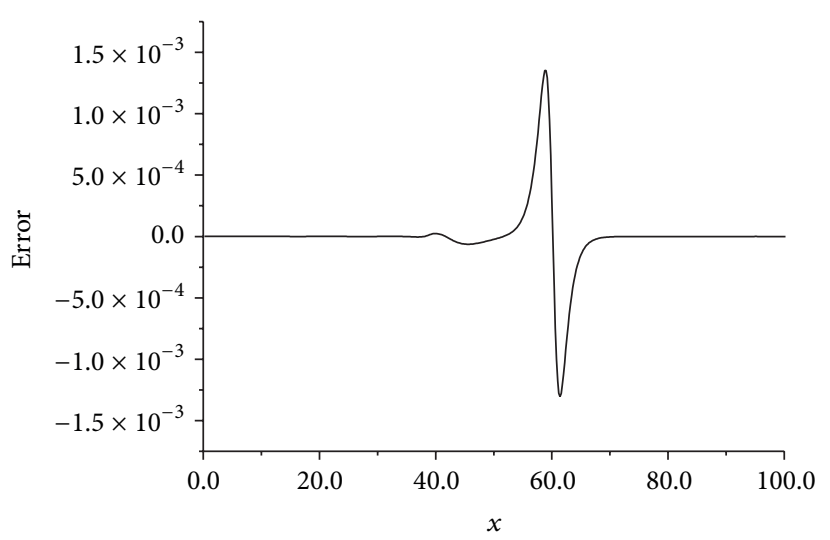

(a)

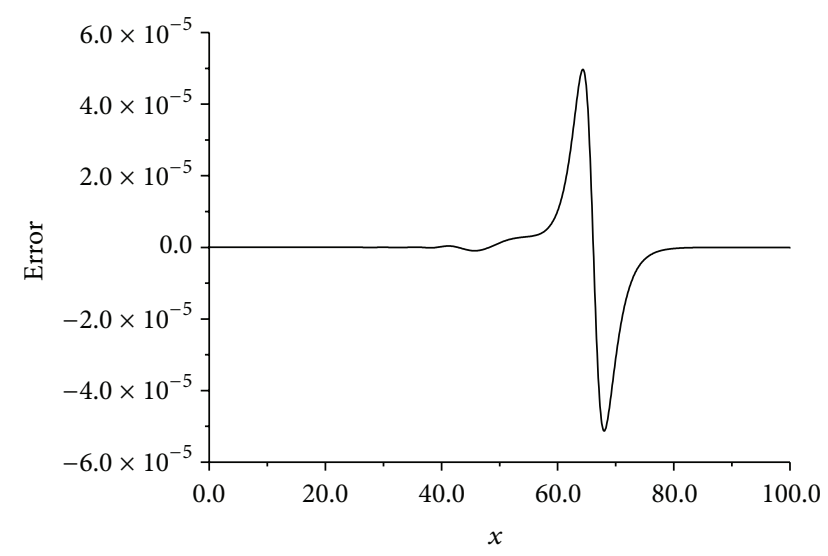

(b)

FIGURE 3: Error with (a) $c=1, h=0.2, \Delta t=0.025, t=10,0 \leq x \leq 100$ and (b) $c=0.3, h=0.1, \Delta t=0.01, t=20,0 \leq x \leq 100$.

TABLE 8: Invariants of MRLW equation using the Maxwellian initial condition.

\begin{tabular}{lcccccccc}
\hline$t$ & $\mu$ & $I_{1}$ & $I_{2}$ & $I_{3}$ & $\mu$ & $I_{1}$ & $I_{2}$ \\
\hline 0 & & 1.7724809 & 1.3786628 & 0.7609109 & & 1.7724809 & 1.2721327 & 0.8674410 \\
3 & & 1.7725930 & 1.4731551 & 0.6664186 & & 1.7753954 & 1.4487372 & 0.6908365 \\
6 & 0.1 & 1.7725929 & 1.4727329 & 0.6668408 & 0.015 & 1.7770146 & 1.4518203 & 0.6877534 \\
9 & & 1.7725935 & 1.4725552 & 0.6670185 & & 1.7786793 & 1.4534252 & 0.6861485 \\
12 & & 1.7725942 & 1.4724619 & 0.6671118 & & 1.7804784 & 1.4552349 & 0.6843388 \\
15 & & 1.77259499 & 1.4724042 & 0.6671695 & & 1.7824228 & 1.4573443 \\
0 & & 1.7724809 & 1.2658662 & 0.8737075 & & 1.7724809 & 1.3034651 & 0.6822294 \\
3 & & 1.7796208 & 1.4490883 & 0.6904854 & & 1.7730165 & 1.4543782 & 0.6851953 \\
6 & 0.01 & 1.7863091 & 1.4571676 & 0.6824061 & 0.04 & 1.7730562 & 1.4540759 & 0.6854978 \\
9 & & 1.7952456 & 1.4658449 & 0.6737288 & & 1.7730962 & 1.4538204 & 0.6857534 \\
12 & & 1.8089221 & 1.4802019 & 0.6593718 & & 1.7731365 & 1.4537359 & 0.6858378 \\
15 & & 1.8364987 & 1.5120445 & 0.6275292 & & 1.7731770 & 1.4537234 & 0.6858503 \\
\hline
\end{tabular}

at different time levels. It is observed that the solitary wave moves to the right with constant velocity and amplitude. At $t=0$, the amplitude is 1.0 which is located at $x=40$, while it is 0.999950 at $t=10$ located at $x=60$. The absolute difference in amplitudes at times $t=0$ and $t=10$ is found to be $5 \times 10^{-5}$, so there is a little change between the amplitudes.

Secondly, we have taken the parameters $\mu=1, c=$ $0.3, h=0.1, k=0.01$ and $x_{0}=40$ with range $[0,100]$ to enable comparison with $[6,30,32,34-36]$. So the solitary wave has amplitude 0.547723 . Simulations are run up to time $t=20$. Error norms $L_{2}$ and $L_{\infty}$ and conserved quantities are tabulated in Table 3 , together with the results obtained in $[6,30,32,34-36]$. These results show high degree of accuracy and efficiency of the method. The invariants $I_{1}, I_{2}$, and $I_{3}$ have changed by less than $0.001 \times 10^{-3} \%, 0.189 \times 10^{-3} \%$, and $1.656 \times 10^{-3} \%$ percent, respectively. Moreover, the variation of the invariants $I_{2}$ and $I_{3}$ from the initial variants is less than $2.5 \times 10^{-6}$ and $2.6 \times 10^{-6}$, respectively, whereas the changes in the invariant $I_{1}$ approach to zero throughout the interval. The perspective views of traveling solitary wave at different time levels are shown in Figure 2. The distributions of the errors at time $t=10$ and $t=20$ are shown graphically for solitary waves amplitudes 1 and 0.3 in Figure 3 . It is seen that the maximum errors are close to the tip of the solitary waves and between $-6 \times 10^{-3}$ and $6 \times 10^{-3},-2 \times 10^{-4}$, and $2 \times 10^{-4}$, respectively. The CPU times for an Intel(R) Core i5, $2.53 \mathrm{GHz}$, are also given in Tables 4 and 5 .

3.2. Interaction of Two Solitary Waves. As a second problem, interaction of two well separated solitary waves having different amplitudes and traveling in the same direction is considered by using the initial condition

$$
U(x, 0)=\sum_{j=1}^{2} A_{j} \operatorname{sech}\left(p_{j}\left[x-x_{j}\right]\right),
$$

where $A_{j}=\sqrt{c_{j}}, p_{j}=\sqrt{c_{j} / \mu\left(c_{j}+1\right)}, j=1,2$, and $c_{j}$ and $x_{j}$ are arbitrary constants. The analytical values of the invariants can be found as [29]

$$
I_{1}=\sum_{j=1}^{2} \frac{\pi \sqrt{c_{j}}}{p_{j}}=11.467698,
$$



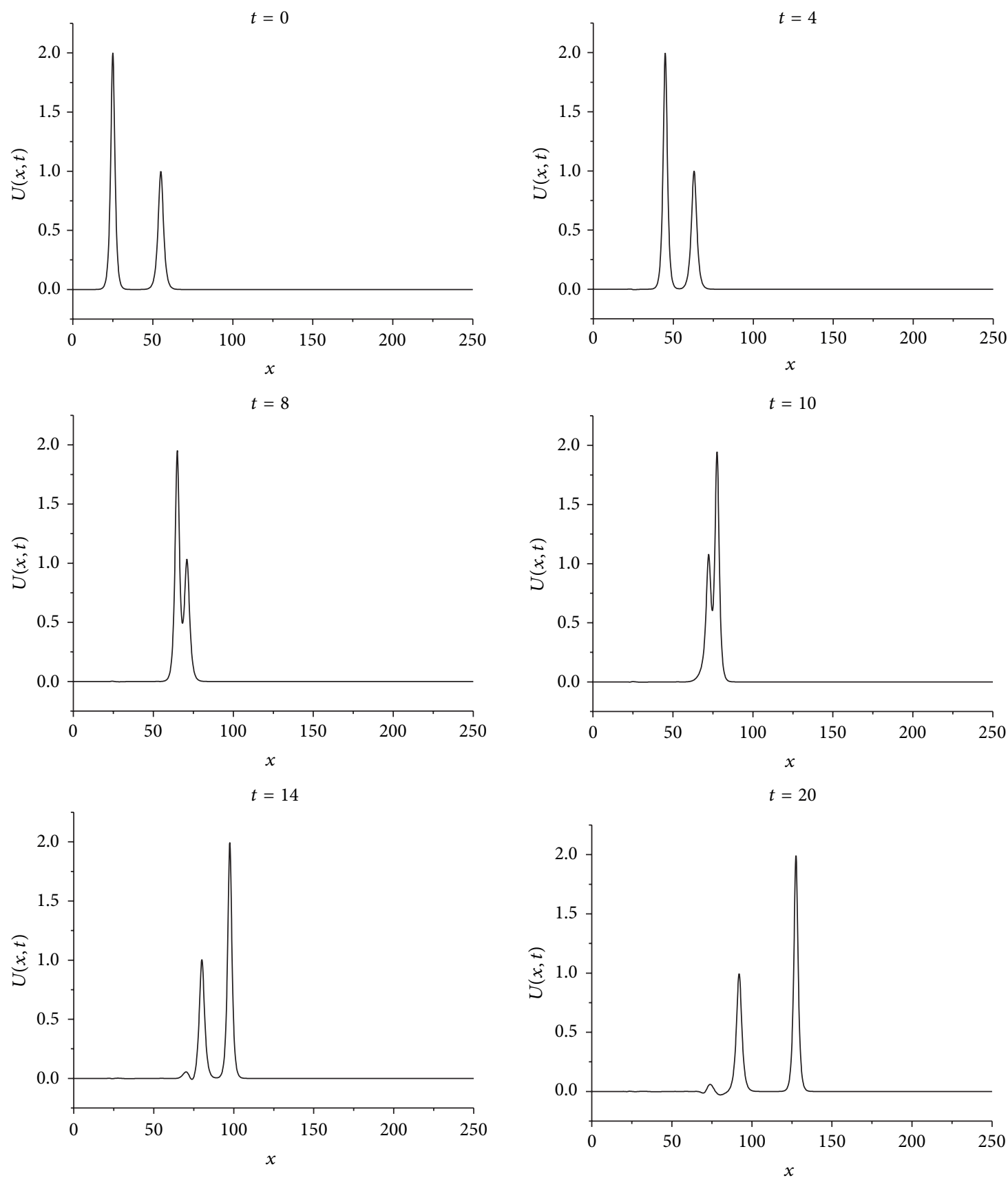

FIGURE 4: Interaction of two solitary waves with $t=0,4,8,10,14$, and 20.

$$
\begin{aligned}
& I_{2}=\sum_{j=1}^{2}\left(\frac{2 c_{j}}{p_{j}}+\frac{2 \mu p_{j} c_{j}}{3}\right)=14.629243, \\
& I_{3}=\sum_{j=1}^{2}\left(\frac{4 c_{j}^{2}}{3 p_{j}}-\frac{2 \mu p_{j} c_{j}}{3}\right)=22.880466 .
\end{aligned}
$$

We have studied the interaction of two positive solitary waves having the parameters $\mu=1, h=0.2, k=0.025, c_{1}=$ $4, c_{2}=1, x_{1}=25, x_{2}=55$ through the interval $0 \leq x \leq 250$ to coincide with those used by $[6,30,34-36]$. The simulations are maintained up to $t=20$. Constant values $I_{1}, I_{2}$, and $I_{3}$ at various time steps together with equivalent results for the previous methods are shown in Table 6. It is seen that the numerical values of the invariants remain almost constant during the computer run. The interaction of two solitary waves is shown in Figure 4. It can be seen from the figure that, at $t=0$, the wave with larger amplitude is to the left of the second wave with smaller amplitude. Since the taller wave moves faster than the shorter one, it catches up and collides with the shorter one at $t=8$ and then moves away from the 

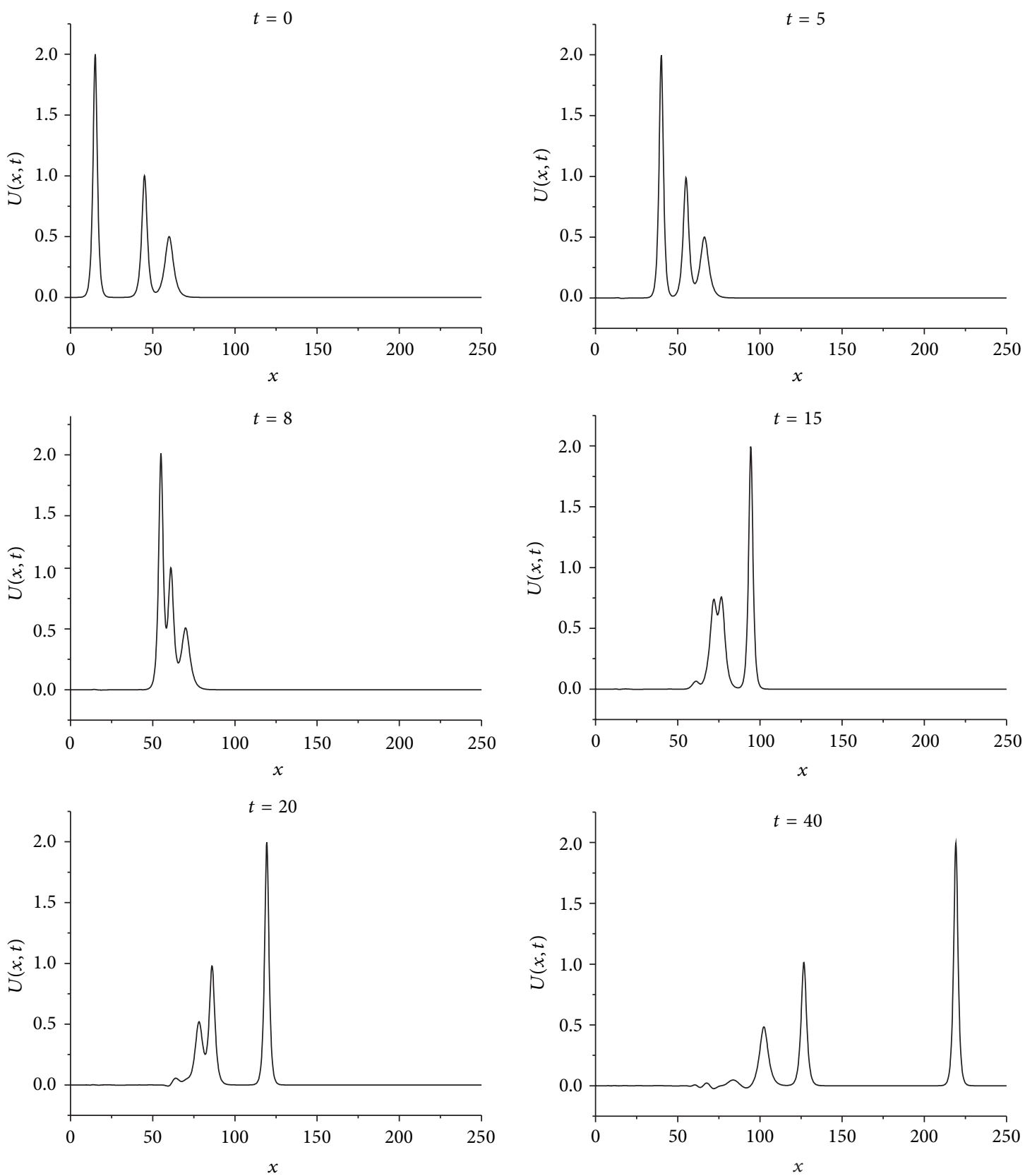

FIGURE 5: Interaction of three solitary waves with $t=0,5,8,15,20$, and 40 .

shorter one as time increases. At $t=20$, the amplitude of larger waves is 2.001102 at the point $x=127.4$, whereas the amplitude of the smaller one is 0.996403 at the point $x=92$. It is found that the absolute difference in amplitude is $3.59 \times 10^{-3}$ for the smaller wave and $1.10 \times 10^{-3}$ for the larger wave for this case.

3.3. Interaction of Three Solitary Waves. As a third problem, interaction of three solitary waves having different amplitudes and travelling in the same direction is studied. We consider (3) with initial conditions given by

$$
U(x, 0)=\sum_{j=1}^{3} A_{j} \operatorname{sech}\left(p_{j}\left[x-x_{j}\right]\right),
$$

where $A_{j}=\sqrt{c_{j}}, p_{j}=\sqrt{c_{j} / \mu\left(c_{j}+1\right)}, j=1,2,3, c_{j}$ and $x_{j}$ are arbitrary constants. For this problem the analytical values of the invariants are found from (32) as

$$
\begin{aligned}
& I_{1}=\sum_{j=1}^{3} \frac{\pi \sqrt{c_{j}}}{p_{j}}=14.9801, \\
& I_{2}=\sum_{j=1}^{3}\left(\frac{2 c_{j}}{p_{j}}+\frac{2 \mu p_{j} c_{j}}{3}\right)=15.8218, \\
& I_{3}=\sum_{j=1}^{3}\left(\frac{4 c_{j}^{2}}{3 p_{j}}-\frac{2 \mu p_{j} c_{j}}{3}\right)=22.9923 .
\end{aligned}
$$




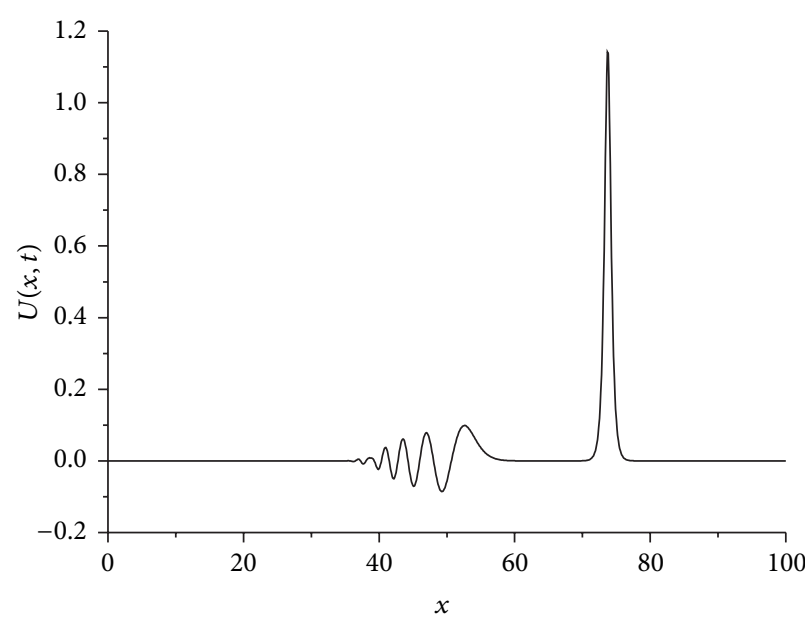

(a)

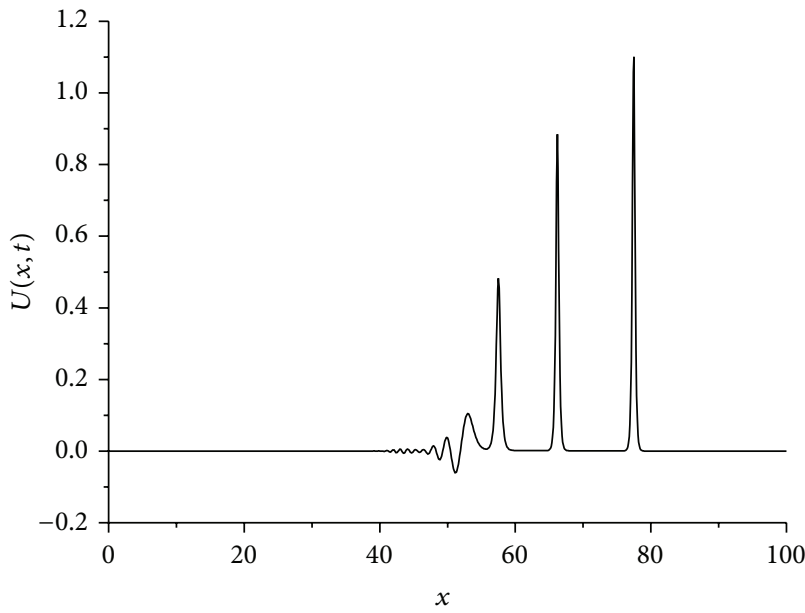

(c)

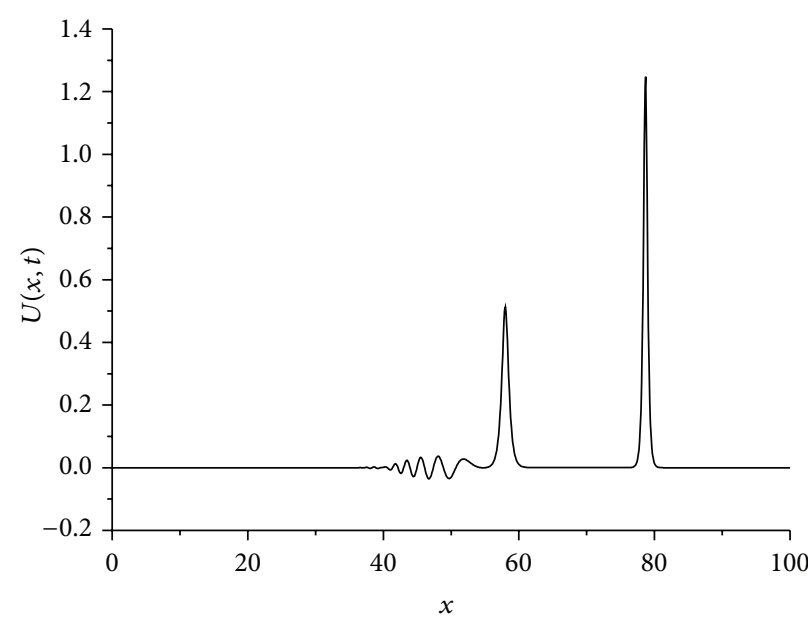

(b)

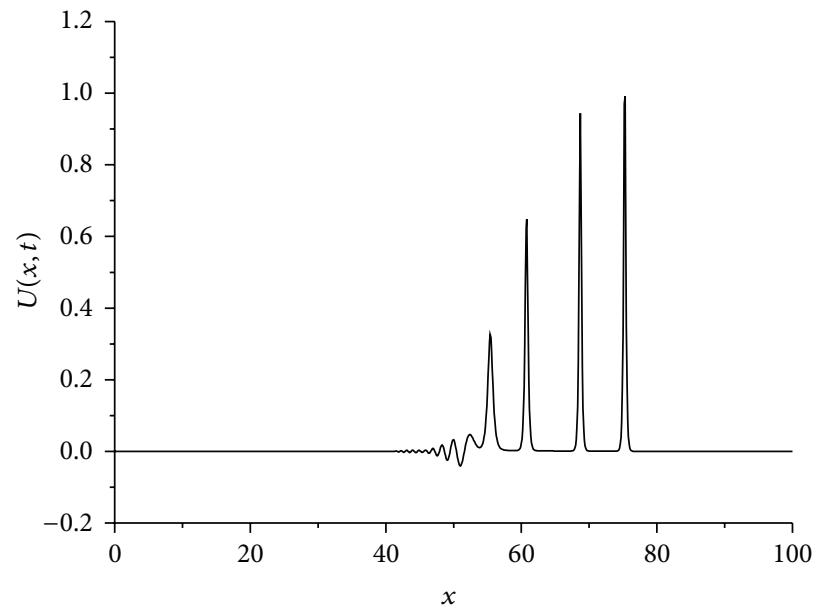

(d)

FIGURE 6: Maxwellian initial condition at $t=14.5$ with (a) $\mu=0.1$, (b) $\mu=0.015$, (c) $\mu=0.01$, and (d) $\mu=0.04$.

In order to be able to compare with the previous works, computations are performed for the parameters $\mu=1, h=$ $0.2, k=0.025, c_{1}=4, c_{2}=1, c_{3}=0.25, x_{1}=15, x_{2}=$ $45, x_{3}=60$ over the interval $0 \leq x \leq 250$. The simulation is run from $t=0$ to $t=45$. Table 7 shows a comparison of the values of the invariants obtained by the present method with those obtained in [30, 34-36]. It is evident from the table that the obtained values of the invariants remain almost constant during the computer run which are all in good agreement with their analytical values given by (36). Figure 5 depicts the interaction of three solitary waves at different times. It is observed from Figure 5 that interaction started about time $t=10$, overlapping processes occurred between time $t=15$ and $t=40$, and waves started to resume their original shapes after the time $t=40$.

3.4. The Maxwellian Initial Condition. As our last problem, the development of the Maxwellian initial condition,

$$
U(x, 0)=\exp \left(-(x-40)^{2}\right)
$$

into a train of solitary waves is considered. For the Maxwellian initial condition, behavior of the solution depends on the values of $\mu$. So we take $\mu=0.1, \mu=$ $0.04, \mu=0.015$, and $\mu=0.01$. The numerical values of the invariants quantities during the simulations are given in Table 8 . For $\mu=0.1$, only a single soliton occurred as depicted in Figure 6(a). When $\mu=0.015$ and $\mu=0.01$, two and three stable solitons occurred, respectively, as depicted in Figures $6(\mathrm{~b})$ and $6(\mathrm{c})$. For $\mu=0.04$, Maxwellian initial condition has decayed into four solitary waves as depicted in Figure 6(d). As is seen, when $\mu$ is reduced, more solitary waves occurred. All figures are drawn up at time $t=14.5$. The peaks of the well developed wave lie on a straight line so that their velocities are linearly dependent on their amplitudes and also we observe a small oscillating tail appearing behind the last wave in all Maxwellian figures.

\section{Conclusion}

In this paper, a numerical treatment of the MRLW equation has been introduced using septic B-spline collocation finite 
element method. The nonlinear term in the equation has been linearized by using a form given in the paper [37]. To examine the performance of the scheme, four test problems have been studied. The performance and accuracy of the method have been tested by calculating the error norms $L_{2}$ and $L_{\infty}$ and the invariant quantities $I_{1}, I_{2}$, and $I_{3}$. Linear stability analysis proved that the present scheme is unconditionally stable. The experimental results of the algorithm are much satisfactory in comparison with the previous results. Our method can successfully be used to model the motion and interaction of the solitary waves. Thus, we can assert that our scheme is efficient and reliable for obtaining the numerical solutions of the other physically important nonlinear partial differential equations.

\section{Conflict of Interests}

The authors declare that there is no conflict of interests regarding the publication of this paper.

\section{Acknowledgment}

The authors wish to thank the reviewers for their careful reading and making several useful comments improving the presented paper.

\section{References}

[1] D. H. Peregrine, "Calculations of the development of an undular bore," Journal of Fluid Mechanics, vol. 25, pp. 321-330, 1966.

[2] D. H. Peregrine, "Long waves on a beac," Journal of Fluid Mechanics, vol. 27, pp. 815-827, 1967.

[3] L. Zhang, "A finite difference scheme for generalized regularized long-wave equation," Applied Mathematics and Computation, vol. 168, no. 2, pp. 962-972, 2005.

[4] D. Kaya, "A numerical simulation of solitary-wave solutions of the generalized regularized long-wave equation," Applied Mathematics and Computation, vol. 149, no. 3, pp. 833-841, 2004.

[5] J. I. Ramos, "Solitary wave interactions of the GRLW equation," Chaos, Solitons and Fractals, vol. 33, no. 2, pp. 479-491, 2007.

[6] T. Roshan, "A Petrov-Galerkin method for solving the generalized regularized long wave (GRLW) equation," Computers \& Mathematics with Applications, vol. 63, no. 5, pp. 943-956, 2012.

[7] R. Mokhtari and M. Mohammadi, "Numerical solution of GRLW equation using Sinc-collocation method," Computer Physics Communications, vol. 181, no. 7, pp. 1266-1274, 2010.

[8] T. B. Benjamin, J. L. Bona, and J. J. Mahony, "Model equations for long waves in nonlinear dispersive systems," Philosophical Transactions of the Royal Society of London A: Mathematical and Physical Sciences, vol. 272, no. 1220, pp. 47-78, 1972.

[9] Q. S. Chang, G. B. Wang, and B. L. Guo, "Conservative scheme for a model of nonlinear dispersive waves and its solitary waves induced by boundary motion," Journal of Computational Physics, vol. 93, no. 2, pp. 360-375, 1991.

[10] L. R. T. Gardner and G. A. Gardner, "Solitary waves of the regularised long-wave equation," Journal of Computational Physics, vol. 91, no. 2, pp. 441-459, 1990.

[11] L. R. T. Gardner, G. A. Gardner, and A. Dogan, "A least-squares finite element scheme for the RLW equation," Communications in Numerical Methods in Engineering, vol. 12, no. 11, pp.795-804, 1996.

[12] L. R. T. Gardner, G. A. Gardner, and I. Dag, "A B-spline finite element method for the regularized long wave equation," Communications in Numerical Methods in Engineering, vol. 11, no. 1, pp. 59-68, 1995.

[13] M. E. Alexander and J. L. Morris, "Galerkin methods applied to some model equations for non-linear dispersive waves," Journal of Computational Physics, vol. 30, no. 3, pp. 428-451, 1979.

[14] J. M. Sanz-Serna and I. Christie, "Petrov-Galerkin methods for nonlinear dispersive waves," Journal of Computational Physics, vol. 39, no. 1, pp. 94-102, 1981.

[15] A. Dogan, "Numerical solution of RLW equation using linear finite elements within Galerkin's method," Applied Mathematical Modelling, vol. 26, no. 7, pp. 771-783, 2002.

[16] A. Esen and S. Kutluay, "Application of a lumped Galerkin method to the regularized long wave equation," Applied Mathematics and Computation, vol. 174, no. 2, pp. 833-845, 2006.

[17] A. A. Soliman and K. R. Raslan, "Collocation method using quadratic B-spline for the RLW equation," International Journal of Computer Mathematics, vol. 78, no. 3, pp. 399-412, 2001.

[18] A. A. Soliman and M. H. Hussien, "Collocation solution for RLW equation with septic spline," Applied Mathematics and Computation, vol. 161, no. 2, pp. 623-636, 2005.

[19] K. R. Raslan, "A computational method for the regularized long wave (RLW) equation," Applied Mathematics and Computation, vol. 167, no. 2, pp. 1101-1118, 2005.

[20] B. Saka, I. Dag, and A. Dogan, "Galerkin method for the numerical solution of the RLW equation using quadratic Bsplines," International Journal of Computer Mathematics, vol. 81, no. 6, pp. 727-739, 2004.

[21] İ. Dağ, B. Saka, and D. Irk, "Application of cubic B-splines for numerical solution of the RLW equation," Applied Mathematics and Computation, vol. 159, no. 2, pp. 373-389, 2004.

[22] I. Dağ and M. Naci Özer, "Approximation of the RLW equation by the least square cubic B-spline finite element method," Applied Mathematical Modelling, vol. 25, no. 3, pp. 221-231, 2001.

[23] S. I. Zaki, "Solitary waves of the splitted RLW equation," Computer Physics Communications, vol. 138, no. 1, pp. 80-91, 2001.

[24] J. C. Eilbeck and G. R. McGuire, "Numerical study of the regularized long-wave equation, II. Interaction of solitary waves," Journal of Computational Physics, vol. 23, no. 1, pp. 63-73, 1977.

[25] P. C. Jain, R. Shankar, and T. V. Singh, "Numerical solution of regularized long-wave equation," Communications in Numerical Methods in Engineering, vol. 9, no. 7, pp. 579-586, 1993.

[26] D. Bhardwaj and R. Shankar, "A computational method for regularized long wave equation," Computers \& Mathematics with Applications, vol. 40, no. 12, pp. 1397-1404, 2000.

[27] B. Y. Guo and W. M. Cao, “The Fourier pseudospectral method with a restrain operator for the RLW equation," Journal of Computational Physics, vol. 74, no. 1, pp. 110-126, 1988.

[28] S. Islam, F. Haq, and A. Ali, "A meshfree method for the numerical solution of the RLW equation," Journal of Computational and Applied Mathematics, vol. 223, no. 2, pp. 997-1012, 2009.

[29] L. R. T. Gardner, G. A. Gardner, and F. A. a. Ayoub, "Approximations of solitary waves of the MRLW equation by $B$-spline finite elements," The Arabian Journal for Science and Engineering A: Sciences, vol. 22, no. 2, pp. 183-193, 1997.

[30] A. K. Khalifa, K. R. Raslan, and H. M. Alzubaidi, "A collocation method with cubic B-splines for solving the MRLW equation," 
Journal of Computational and Applied Mathematics, vol. 212, no. 2, pp. 406-418, 2008.

[31] A. K. Khalifa, K. R. Raslan, and H. M. Alzubaidi, "A finite difference scheme for the MRLW and solitary wave interactions," Applied Mathematics and Computation, vol. 189, no. 1, pp. 346354, 2007.

[32] K. R. Raslan, "Numerical study of the modified regularized long wave (MRLW) equation," Chaos, Solitons and Fractals, vol. 42, no. 3, pp. 1845-1853, 2009.

[33] K. R. Raslan and S. M. Hassan, "Solitary waves for the MRLW equation," Applied Mathematics Letters, vol. 22, no. 7, pp. 984989, 2009.

[34] F. Haq, S. Islam, and I. A. Tirmizi, "A numerical technique for solution of the MRLW equation using quartic B-splines," Applied Mathematical Modelling, vol. 34, no. 12, pp. 4151-4160, 2010.

[35] A. Ali, Mesh free collocation method for numerical solution of initial-boundary value problems using radial basis functions [Ph.D. thesis], 2009.

[36] S. B. G. Karakoc, N. M. Yagmurlu, and Y. Ucar, "Numerical approximation to a solution of the modified regularized long wave equation using quintic B-splines," Boundary Value Problems, vol. 2013, article 27, 2013.

[37] S. G. Rubin and R. A. Graves, "Cubic spline approximation for problems in fluid mechanics," TR R-436, Nasa, Washington, DC, USA, 1975.

[38] S. B. G. Karakoc and T. Geyikli, "Petrov-Galerkin finite element method for solving the MRLW equation," Mathematical Sciences, vol. 7, article 25, 2013.

[39] Y. Khan, R. Taghipour, M. Falahian, and A. Nikkar, "A new approach to modified regularized long wave equation," Neural Computing and Applications, vol. 23, pp. 1335-1341, 2013.

[40] P. M. Prenter, Splines and Variational Methods, John Wiley \& Sons, New York, NY. USA, 1975.

[41] P. J. Olver, "Euler operators and conservation laws of the BBM equation," Mathematical Proceedings of the Cambridge Philosophical Society, vol. 85, no. 1, pp. 143-160, 1979. 


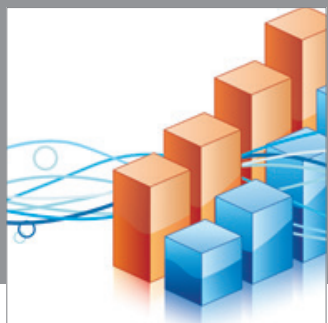

Advances in

Operations Research

mansans

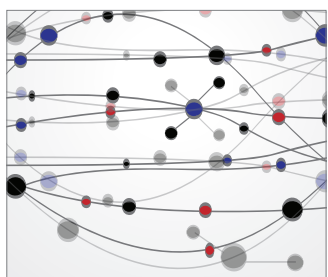

The Scientific World Journal
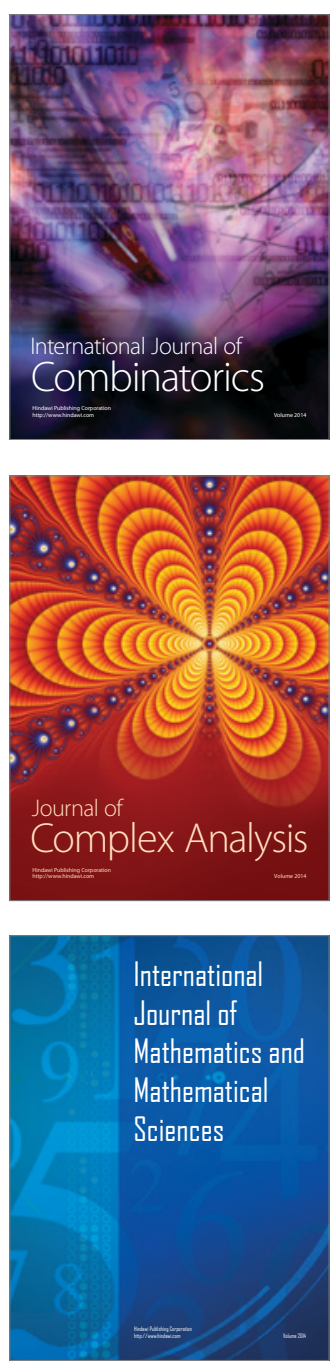
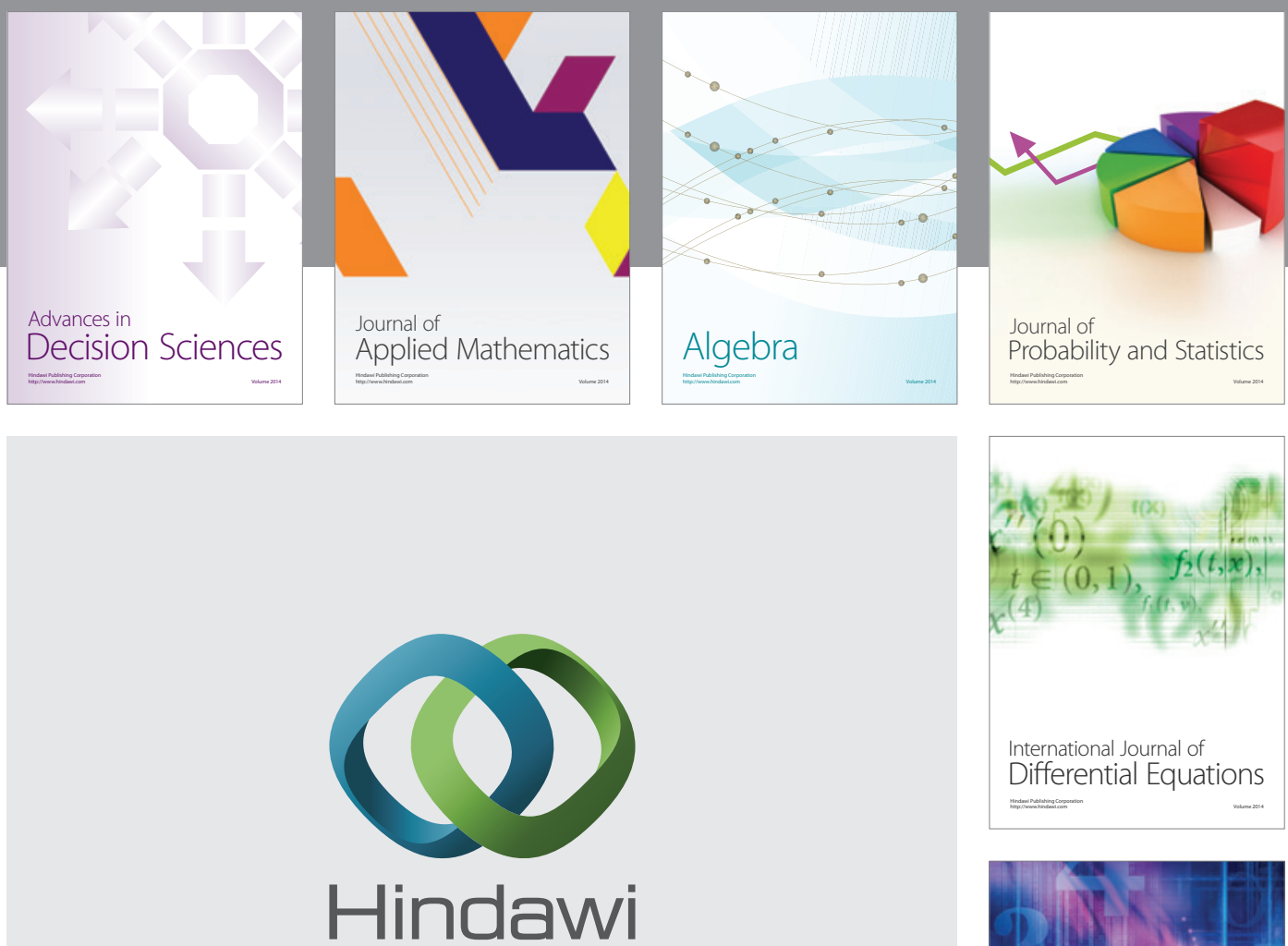

Submit your manuscripts at http://www.hindawi.com
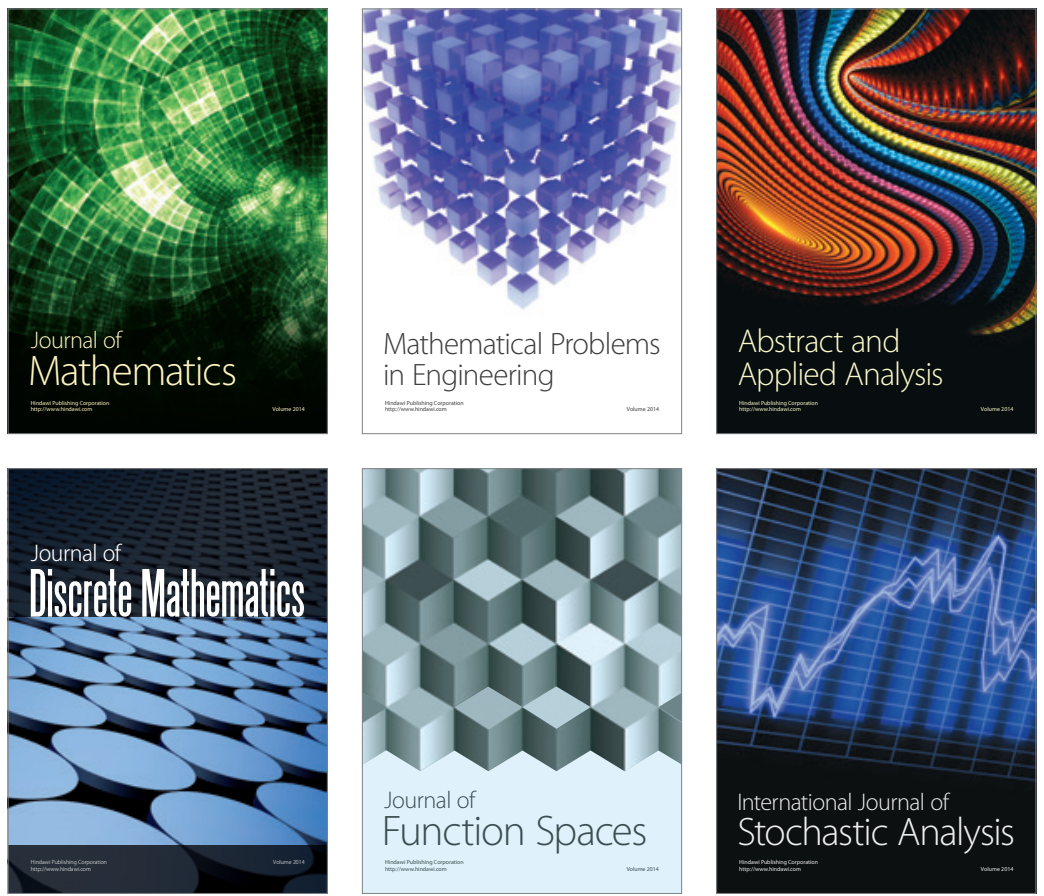

Journal of

Function Spaces

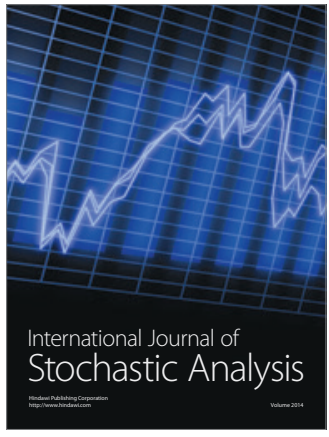

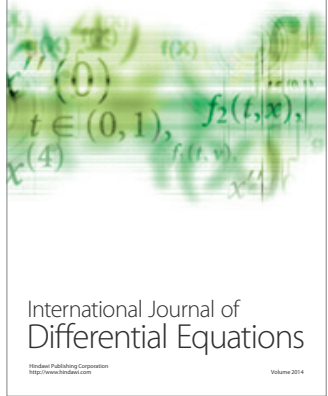
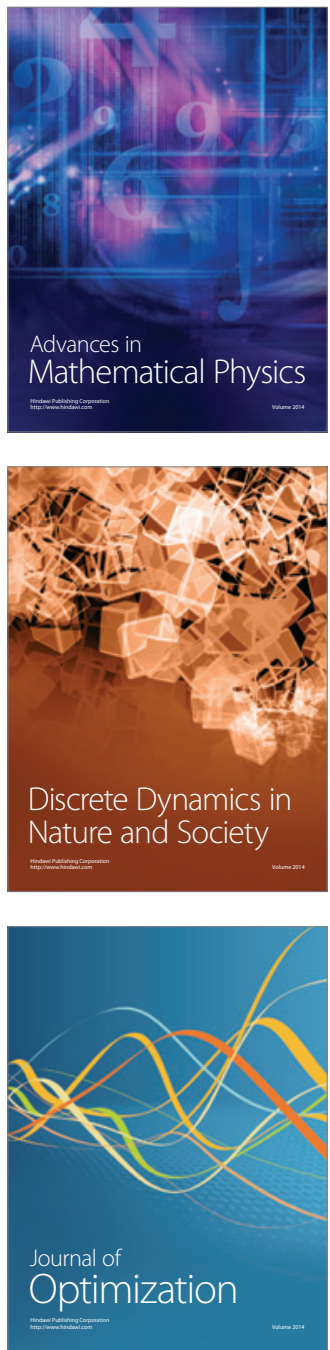